OPEN ACCESS

Edited by:

Yanqiong Zhang,

China Academy of Chinese Medical

Sciences, China

Reviewed by:

Gangjun Du,

Henan University, China

Yanfang Zheng,

Fujian University of Traditional Chinese

Medicine, China

*Correspondence:

Xiaohong Liu

rsclxh@gzucm.edu.cn

Linlin Lu

Illu@gzucm.edu.cn

${ }^{t}$ These authors have contributed equally to this work

Specialty section:

This article was submitted to

Ethnopharmacology,

a section of the journal

Frontiers in Pharmacology

Received: 16 June 2020 Accepted: 05 August 2020 Published: 26 August 2020

Citation:

Huang Q, Feng L, Li H, Zheng L, Qi X, Wang Y, Feng $Q$, Liu Z, Liu X and Lu L (2020) Jian-Pi-Bu-Xue-Formula Alleviates Cyclophosphamide-Induced Myelosuppression via Up-Regulating NRF2/HO1/NQO1 Signaling.

Front. Pharmacol. 11:1302. doi: 10.3389/fphar.2020.01302

\section{Jian-Pi-Bu-Xue-Formula Alleviates Cyclophosphamide-Induced Myelosuppression via Up-Regulating NRF2/H01/NQ01 Signaling}

\author{
Qiuju Huang ${ }^{1,2 \dagger}$, Lizhi Feng ${ }^{1,3 \dagger}$, Hang $L^{1,3}{ }^{1,3}$,iang Zheng ${ }^{1}$, Xiaoxiao Qi ${ }^{1}$, Ying Wang ${ }^{1}$, \\ Qian Feng ${ }^{1}$, Zhongqiu Liu ${ }^{1}$, Xiaohong Liu ${ }^{1,3^{*}}$ and Linlin Lu ${ }^{1 *}$
}

1 Joint Laboratory for Translational Cancer Research of Chinese Medicine of the Ministry of Education of the People's Republic of China, International Institute for Translational Chinese Medicine, Guangzhou University of Chinese Medicine, Guangzhou, China, ${ }^{2}$ School of Basic Medical Sciences, Guangzhou University of Chinese Medicine, Guangzhou, China, ${ }^{3}$ Department of Respiratory Medicine, The First Affiliated Hospital of Guangzhou University of Chinese Medicine, Guangzhou, China

Jian-pi-bu-xue-formula (JPBXF), a TCM formula composed of twelve Chinese medicinal herbs, has been used in clinic to ease patients' state of weakness and fatigue especially after receiving anti-tumor chemotherapy in China. The lack of the phytochemical characterization, detail therapeutic evaluation and mechanism of JPBXF remains the main limitation for its spreading. In this study, we systematically evaluated the effectiveness and underline mechanism of JPBXF on cyclophosphamide (CTX)-induced myelosuppression and identified the main constituents of JPBXF aqueous extract. JPBXF treatments reversed CTX-induced myelosuppression through increasing the number of haematopoietic stem cells (HSCs) and expression of C-kit in bone marrow cells. Simultaneously, JPBXF treatments alleviated CTX-induced blood cells reduction by increasing numbers of RBCs and WBCs and levels of GM-CSF, TPO and EPO in plasma. JPBXF treatments reduced CTX-induced immunosuppression by increasing expressions of CD3, CD4, and CD8a in PBMCs, and recovering structure damages of thymus and spleen. Moreover, JPBXF notably increased the expression of NRF2 compared with CTX group, and subsequently up-regulated HO1 and NQO1 both in mRNA and protein levels. In addition, eighteen compounds were recognized from JPBXF aqueous extract and the potential targets of the identified compounds were predicted. Overall, JPBXF can greatly reverse CTX-induced myelosuppression in C57BL/6 mice, especially in improving the blood and immune function through activating NRF2/HO1/ NQO1 signaling pathway, which provides a reliable reference for JPBXF application in clinical. By recognizing eighteen compounds in JPBXF aqueous extract and predicting the underline mechanisms of the identified compounds, our study would provide theoretical guidance for further research of JPBXF.

Keywords: Jian-pi-bu-xue-formula, cyclophosphamide, complementary therapy, myelosuppression, NRF2 


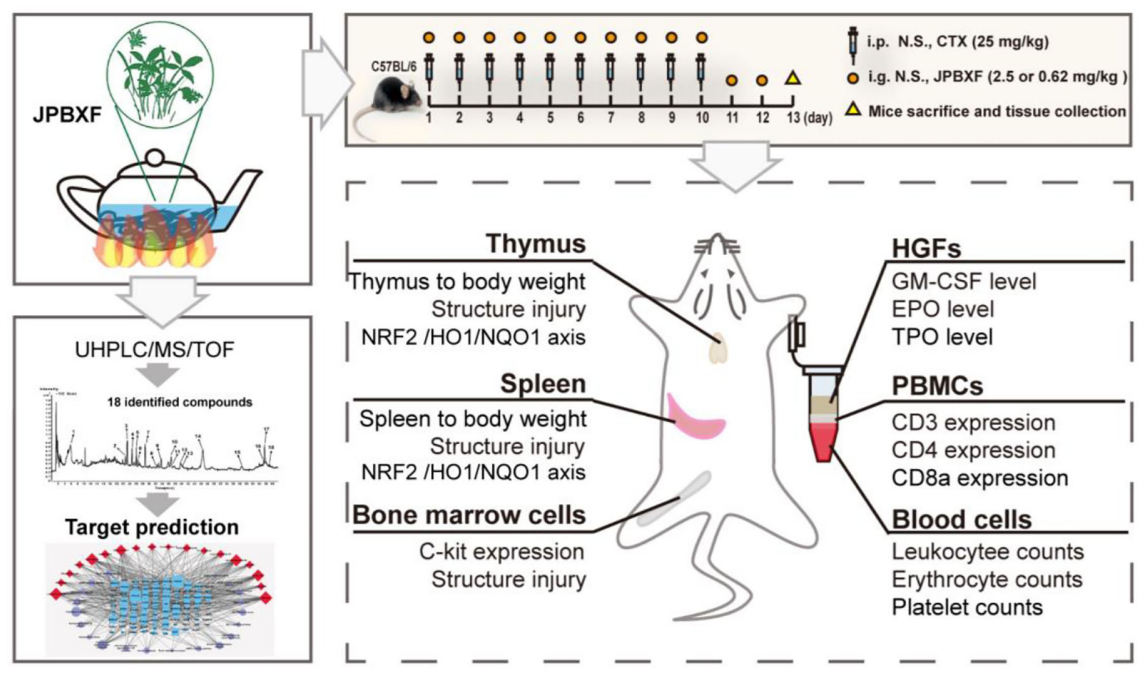

\section{INTRODUCTION}

Cancer, an aggressive malignancy, approximately causes 4,285,033 new cases and 2,865,147 cancer-related deaths annually in China (Bray et al., 2018). The conventional therapies for cancer treatment include chemotherapy, radiotherapy and surgery. Among these treatments, chemotherapy acts as a practical method and was widely used to kill cancer cells by impeding their growth and reproduction (Potti et al., 2011). However, most of chemotherapeutic agents pose many side effects including myelosuppression, immunosuppression, gastrointestinal reaction and hepatic or renal toxicity, that greatly threaten patients' quality of life, reduce treatments' efficiency, and furthermore lead to termination of ongoing useful therapy (Tachi et al., 2015). For instance, cyclophosphamide (CTX), a common anti-cancer chemotherapeutic agent, is often used alone or in combination with platinum and paclitaxel as standard first-line therapy (Handolias et al., 2016). Myelosuppression, the most common side effect of CTX characterized by decrease of stem and progenitor cells in bone marrow, often arises complications such as neutropenia, anemia and thrombocytopenia (Carey, 2003; Neboh and Ufelle, 2015). It has proof that NRF2 deficiency deteriorated CTX-induced myelosuppression, while activation of NRF2 mitigates ionizing CTX -induced myelosuppression (Que et al., 2016). The up-regulation of HO1 and NQO1 could resist to CTX-induced bone marrow suppression, oxidative stress,

Abbreviations: BATMAN-TCM, Bioinformatics Analysis Tool for Molecular mechANism of Traditional Chinese Medicine; CTX, cyclophosphamide; DAVID, Database for Annotation, Visualization and Integrated Discovery; ELISA, enzyme-linked immunosorbent assay; EPO, erythropoietin; G-CSF, granulocyte colony-stimulating factor; GM-CSF, granulocyte macrophage colony-stimulating factor; JPBXF, Jian-pi-bu-xue-formula; PBMC, peripheral blood mononuclear cell; PPI, protein-protein interaction; TCM, traditional Chinese medicine; TPO, thrombopoietin. inflammation and apoptosis (Chen et al., 2014; HAS et al., 2019). Therefore, methods, which could prevent or eliminate chemotherapy-induced side effects through regulating NRF2 pathway, have great significance for patients struggling against cancer.

Complementary medicine, forms of treatment that used along with standard medical treatments, is common among cancer patients to help lessen some side effects of cancer treatment (Hubner et al., 2015). In clinical, hematopoietic growth factors (HGFs) including granulocyte colony-stimulating factor (G$\mathrm{CSF}$ ) and granulocyte macrophage colony-stimulating factor (GM-CSF), are effective complementary medicines for therapy to alleviate chemotherapy-induced myelosuppression (Miller and Steinbach, 2014). Unfortunately, G-CSF and GM-CSF can also accelerate growth of cancer cells (Gutschalk et al., 2006). Therefore, new approaches on finding more effective and safe drugs for preventing and treating chemotherapy-induced myelosuppression are necessary. Traditional Chinese medicine (TCM) complementary treatment has been reported to alleviate chemotherapy induced side effects and improve quality of life (Zhang et al., 2007; Konkimalla and Efferth, 2008). For instance, Ipomoea obscura (L.) Ker Gawl. extract could reduce CTXinduced myelosuppression, improve white blood cell counts and recover bone marrow cellularity (Hamsa and Kuttan, 2010). Sip-jeon-dea-bo-tang, a traditional herbal medicine, has been reported to prevent the cisplatin-induced reduction of food intake and weight loss (Woo et al., 2016). Considering the limitations of hematopoietic growth factors, finding effective TCMs on alleviating chemotherapy-induced myelosuppression is helpful and meaningful.

Jian-pi-bu-xue-formula (JPBXF), a TCM consisted of twelve Chinese medicinal herbs, is consumed in clinical for strengthening spleen and fortifying the blood in China for decades. According to basic theories of Chinese medicine, 
JPBXF is a combined formula based on Si-Jun-Zi-Tang, Si-WuTang and Dang-Gui-Bu-Xue-Tang without unnecessary drugs, aiming to improve the hematopoiesis effects and make it gently to patients with chemotherapy. JPBXF is composed of monarch drugs Hedysarum multijugum Maxim (Huang-Qi) and Codonopsis pilosulae (Franch.) Nannf. (Codonopsis pilosulae Radix, Dang-Shen), minister drugs including Ficus simplicissima Lour. syn. Ficus hirta Vahl (Wu-zhi-mao-tao), Atractylodes macrocephala Koidz. (Bai-Zhu), Angelica sinensis (Oliv.) Diels (Dang-Gui), and Rehmannia glutinosa (Gaertn.) DC. (Shu-Di-Huang), assistant drugs including Pinellia ternata (Thunb.) Makino syn. Arum ternatum Thunb. (Ban-Xia), Citrus $\times$ aurantium L. syn. Citrus reticulata Blanco (Chen-Pi), Pogostemon cablin (Blanco) Benth. (Huo-Xiang) and Zingiber officinale Roscoe (Sheng-Jiang), and guide drugs Ziziphus jujuba Mill (Da-Zao) and Glycyrrhiza uralensis Fisch (Gan-Cao). In JPBXF, H. multijugum, C. pilosula and R. glutinosa had been reported to enhance immunity in vivo after CTX treatment (Cohen et al., 2002; Wang et al., 2012). A. sinensis has great impacts on promoting hematopoiesis (Gong et al., 2016). Z. jujuba, Z. officinale, A. ternatum, P. cablin, C. aurantium, A. macrocephala and G. uralensis are often used in formulas for antiemetic during chemotherapy (Cohen et al., 2002). Our previous study also showed that JPBXF could improve hematopoiesis of chemotherapy mice by affecting the expression levels of GM-CSF, TPO, and EPO (Feng and Liu, 2015). Although JPBXF has been used as a formula in clinical for decades, the lack of detail therapeutic evaluation and underline mechanism of JPBXF remains the main limitation for its spreading. Furthermore, the phytochemical characterization of JPBXF is still unclear.

In our study, a CTX-induced myelosuppression animal model was established to assess the effectiveness and safety of JPBXF in alleviating chemotherapy-induced myelosuppression. Changes in thymus, spleen, bone marrow, blood cell counts, expressions of T-cell surface markers such as CD3, CD4, and CD8a in peripheral blood mononuclear cells (PBMCs), expression of Ckit in bone marrow cells, as well as levels of GM-CSF, TPO, and EPO in plasma in the mice were systematically evaluated. Meanwhile, effects of JPBXF on NRF2, HO1, and NQO1 expressions in thymus and spleen were also detected. Furthermore, main chemical constituents of JPBXF aqueous extract were identified using UHPLC/MS/TOF and the underline mechanisms of the identified compounds were predicted using bioinformatics analysis.

\section{MATERIAL AND METHODS}

\section{Reagents}

Cyclophosphamide (Pude Pharmaceutical Co., Datong, Shanxi, China) was purchased from the First Affiliated Hospital of Guangzhou University of Chinese Medicine. The anti-CD3 and anti-CD4 antibodies, goat anti-rabbit and anti-mouse secondary antibody horseradish peroxidase conjugate (IgG antibodies) were purchased from Abcam (Cambridge, Massachusett, UK). The antiCD8a antibody and anti-C-kit receptor antibody were purchased from Santa Cruz Biotechnology (Santa Cruz, California, USA). GM-CSF, TPO, and EPO Elisa kits were purchased from R\&D Systems (Minneapolis, MN., USA). All other reagents were from a standard source and were analytical pure grade.

\section{Plant Materials and JPBXF Preparation}

F. simplicissima (30 g), H. multijugum (30 g), C. pilosula (20 g), A. macrocephala (15 g), A. sinensis (6 g), R. glutinosa (10 g), A. ternatum (10 g), C. aurantium (10 g), P. cablin (10 g), $Z$. officinale (6 g), Z. jujuba (10 g) and G. uralensis (10 g) were all purchased from the First Affiliated Hospital of Guangzhou University of Traditional Chinese Medicine (Guangdong, China) and identified by Institute of Chinese Materia Medica, China Academy of Chinese Medical Sciences (Beijing, China). A voucher specimen was deposited at the Laboratory of International Institute for Translational Chinese Medicine, Guangzhou University of Chinese Medicine (Guangzhou, China). The Chinese herbal mixture was immersed in a 8-fold amount of distilled water for $30 \mathrm{~min}$ and gently boiled for $30 \mathrm{~min}$. The extract was collected. The herb residues were boiled again with a 6-fold amount of distilled water for $1 \mathrm{~h}$, and the extract was collected again. Following, the collection extract were combined, filtered with three layers of gauze, and evaporated in $60{ }^{\circ} \mathrm{C}$. After cooling at room temperature, liquid was spared and stored at $4{ }^{\circ} \mathrm{C}$.

\section{JPBXF Chemical Components Analysis}

Characterization of main chemical components in JPBXF was detected by UHPLC/MS/TOF (Agilent Technologies 6540). Chromatographic separation was achieved on a Zorbax C18 column $\left(100 \times 3.0 \mathrm{~mm}^{2}, 1.8 \mu \mathrm{m}\right)$ (Agilent Technologies), with column temperature maintained at $25^{\circ} \mathrm{C}$. The mobile phases consisted of water (A) and acetonitrile (B) using a gradient elution. The flow rate was $0.3 \mathrm{ml} / \mathrm{min}$. The mass spectrometer was operated in negative ion mode. The full scan setting parameters are as follows: capillary voltage, $2.5 \mathrm{kV}$; nozzle voltage, $1,000 \mathrm{~V}$; gas temperature, $290^{\circ} \mathrm{C}$; sheath temperature, $340^{\circ} \mathrm{C}$; sheath gas flow, $11 \mathrm{~L} / \mathrm{min}$; and gas flow, $10 \mathrm{~L} / \mathrm{min}$; fragmentation voltage: $135 \mathrm{~V}$. Self-building database containing mass spectrometry information of reported compounds from each herb was used for compounds matching.

\section{Animals}

Male C57BL/6 mice (4-6 weeks, 18-22 g) were purchased from the Laboratory Animal Center of Guangzhou University of Chinese Medicine (Guangzhou, China; License: SCXK, Guangdong, 2018-0034). The mice were kept in the animal facility in the SPF animal laboratory (License number: SYXK (GZ) 2019-0144) at International Institute for Translational Chinese Medicine, Guangzhou University of Chinese Medicine (Guangzhou, China). The animal experiments were approved by the International Institute for Translational Chinese Medicine Animal Care and Use Committee, Guangzhou University of Chinese Medicine (Guangzhou, China). The mice randomly 
divided into five groups: control, CTX, GM-CSF, high- and lowdose JPBXF. Control group $(n=6)$ received treatments with $0.9 \%$ physiologic saline (i.p.) once a day for 10 days and intragastric administrations with $0.9 \%$ physiologic saline once a day for 12 days. CTX, GM-CSF, JPBXF groups (each with $\mathrm{n}=6$ ) received administrations with CTX (25 mg/kg, i.p.) once a day for 10 days and intragastric administrations with $0.9 \%$ physiologic saline, GM-CSF $(5 \mu \mathrm{g} / \mathrm{kg})$, high- and low-dose of JPBXF $(2.5 \mathrm{~g} / \mathrm{mL}$ and $0.62 \mathrm{~g} / \mathrm{mL}$ ) once a day for 12 days, respectively. The body weights of the mice were recorded every other day. Finally, the mice were sacrificed and the organs (including spleen and thymus) of each mouse were isolated and weighed.

\section{Peripheral Blood Cell Count}

Before the mice were sacrificed, the blood samples $(200 \mu \mathrm{L}$ of each mouse) were collected in heparin-treated tubes by and then stored at $4^{\circ} \mathrm{C}$. After all samples collection, the numbers of leukocyte, platelet, and erythrocyte were counted using SYSMEX XT-1800I (Kobe, Japan).

\section{Isolation of Plasma and PBMCs}

After the mice were sacrificed, the whole blood of each mouse was collected in heparin-treated tubes and then centrifuged at 3,000 rpm for $10 \mathrm{~min}$. The plasma samples were harvested, and stored at $-80^{\circ} \mathrm{C}$. The remaining precipitation was resuspended in PBS (v/v, 1:1), and loaded into the tubes filled with Ficoll (GE Healthcare, Chicago, USA). After centrifuged at $400 \mathrm{~g}$ for $30 \mathrm{~min}$, PBMCs were isolated, and rinsed twice with PBS.

\section{ELISA Assay}

Levels of GM-CSF, TPO, and EPO in plasma were measured by enzyme-linked immunosorbent assay (ELISA), using Quantikine ELISA kits (R\&D Systems, Minneapolis, MN, USA), according to the manufacturer's instructions. Results were expressed as ng/L of plasma.

\section{Flow Cytometry}

After isolated, PBMCs were treated according our previous study (Feng et al., 2016). After incubated with anti-CD3 antibody (1:200), anti-CD4 antibody (1:200), anti-CD8a antibody (1:50), and secondary antibodies (1:200), the PBMCs samples were detected using Flow cytometry (BD Biosciences, San Diego, CA, USA).

\section{H\&E Staining}

Tissues such as thymus, spleens, and femurs were treated and observed according our previous study (Feng et al., 2016). Briefly, after fixed, embedded, and sliced, sections $(4 \mu \mathrm{m})$ of thymus, spleens, and femurs were deparaffinized and rehydrated. Following, hematoxylin and eosin staining were performed. The slices were then observed under a light microscope (Leica DM750, Wetzlar, GER).

\section{Western Blot}

Total proteins of thymus or spleens were extracted using RIPA lysis buffer and phenylmethanesulfonyl fluoride (PMSF), and quantified using Coomassie Brilliant Blue Kit (Bio-Rad, Hercules, CA, USA). Protein samples of each tissue were separated by $10 \%$ SDS-PAGE, transferred onto PVDF membranes, and blocked with $5 \%$ BSA for $1 \mathrm{~h}$. The membranes were incubated with the primary antibody of NRF2 $(1: 1,000)$ at $4{ }^{\circ} \mathrm{C}$ overnight, subsequently, with the corresponding secondary antibodies $(1: 5,000)$ at room temperature for $1 \mathrm{~h}$. $\beta$-actin acted as a loading control. ECL chemiluminescence reagent was applied to detect for fluorescent signals using FluorChem E (Santa Clara, CA, USA). Protein bands were quantified using Quantity One software (Bio-Rad, Hercules, CA, USA).

\section{Real-Time PCR Analysis}

Total RNA of thymus or spleens was isolated through the TRIzol extraction method. Then, RNA reverse transcribed into cDNA according to a reverse transcription kit (TaKaRa, Shiga, Japan). SYBR Green real-time PCR amplification and detection were then performed with an ABI 7500 system (Applied Biosystems, Foster City, USA). $\beta$-actin was regarded as house-keeping gene. Primers are as follows. HO1: 5'-TGATGGCTTCCTTGTA CCATATC-3' and 3'-AGCTCCTCAGGGAAGTAGAG- 5'; NQO1: 5'-GAGAAGAGCCCTGATTGTACTG-3' and 3'-ACC TCCCATCCTCT CTTCTT-5'; $\beta$-actin: 5'-CTGTCCCTGTAT GCCTCTG-3' and 5'-ATGTCACGCAC GATTTCC-3'.

\section{Immunohistochemistry (IHC)}

Thymus and spleens tissues were fixed in $4 \%$ paraformaldehyde, embedded in paraffin. After sliced up, the slices $(4 \mu \mathrm{m})$ were dewaxed, hydrated, and then incubated with natrium citric $(0.01$ $\mathrm{M})$ for antigen retrieval. Following, the slices were rinsed with PBS, and incubated with anti-HO1 and anti-NQO1 overnight at $4{ }^{\circ} \mathrm{C}$. Following steps were performed using the immunostaining kit (BOSTER Biological Technology) based on the manufacturer's instructions.

\section{Target Prediction by Network Pharmacology}

The myelosuppression-related genes were obtained from the GeneCards database (https://www.genecards.org/), and the potential target genes of compounds were predicted using a Bioinformatics Analysis Tool for Molecular mechANism of Traditional Chinese Medicine (BATMAN-TCM) (http://bionet. ncpsb.org/batman-tcm/) and Swiss Target Prediction database (http://www.swisstargetprediction.ch/). Genes obtained from these three databases were analyzed using Venny 2.1 (https:// bioinfogp.cnb.csic.es/tools/venny/index.html). The gene ontology (GO) and Genomes (KEGG) enrichment analyses were performed for the potential targets using the Database for Annotation, Visualization and Integrated Discovery (DAVID) (https://david.ncifcrf.gov/) and the online software Omicshare. The protein-protein interaction (PPI) among these potential targets was constructed using the STRING database (https:// string-db.org/) (Du et al., 2019). The compound-target-pathway network was constructed using Cytoscape 3.7.2 (Zhang et al., 2019). 


\section{Data Analysis}

All data were expressed as mean \pm standard deviation (SD). Significant differences were analyzed by one-way ANOVA followed by LSD test (for more than two groups) by SPSS. Statistical difference was considered significant at $P<0.05$.

\section{RESULTS}

\section{Phytochemical Characterization of JPBXF}

To identify the main constituents of JPBXF aqueous extract, we analyzed the JPBXF aqueous extract using UHPLC/MS/TOF. Eighteen compounds were recognized from JPBXF aqueous extract as shown in Table 1. The typical scaffold backbones for the eighteen compounds are flavonoids, saponins and polyphenols. Among the eighteen compounds, there are thirteen flavonoids, including liquiritin apioside, liquiritin, acteoside, naringin, isoacteoside, hesperidin, isoliquiritin apioside, isoliquiritin, liquiritigenin, calycosin, formononetin, glycycoumarin, and licoisoflavone B. Meanwhile, licoricesaponine A3, licoricesaponine G2 and glycyrrhizic acid are three saponins. Paeonol and 4-gingerol are two polyphenols. According to UHPLC/MS/TOF, liquiritin, acteoside, naringin, hesperidin, glycyrrhizic acid and 4-gingerol were identified with high contents (Figure 1). Three pairs of flavonoid isomers need further identification.

\section{Effects of JPBXF on CTX-Induced Myelosuppression}

To assess the effects of JPBXF on chemotherapy-induced myelosuppression in mice, body weight change and $\mathrm{H} \& \mathrm{E}$ staining of femurs were performed. Although there was no significant change in body weight among each group, CTX treatment induced a slight decrease in body-weight of mice compared with the control group (Figure 2A). However, after treated with JPBXF, the body-weight loss of mice induced by CTX was decreased slightly (Figure 2A). H\&E staining results shown that CTX treatment significantly reduced the number of haematopoietic stem cells (HSCs) in femurs compared with control group. To the contrary, JPBXF treatments increased the number of HSCs compared with that of CTX treatment alone (Figure 2B). To further confirm whether JPBXF treatments could influence the number of HSCs, the expression of C-kit, a marker of HSCs, was detected in bone marrow cells. The results shown that compared with control, the expression of C-kit was markedly decreased by $57.02 \pm 6.16 \%$ in CTX treated bone marrow cells $(P<$ 0.01 , Figure 2C). Meanwhile, $5 \mu \mathrm{g} / \mathrm{kg}$ of GM-CSF and $2.5 \mathrm{~g} / \mathrm{mL}$ of JPBXF treatments increased the expression of C-kit by $35.68 \pm$ $7.82 \%$ and $42.44 \pm 8.71 \%$ respectively compared with that of CTX alone $(P<0.05$, Figure $2 \mathrm{C})$.

\section{JPBXF Alleviated CTX-Induced Blood Cells Reduction}

Along with myelosuppression, CTX treatments often induce blood cells reduction (Hill et al., 2011). To investigate the effects of JPBXF on blood cells, we detected the numbers of WBCs, RBCs, and PLTs and analyzed the secretion levels of hematopoietic growth factors including GM-CSF, EPO, and TPO. The whole blood analysis revealed that compared with control group, the numbers of WBCs and RBCs were significantly decreased after CTX treatments. However, $5 \mu \mathrm{g} / \mathrm{kg}$ of GM-CSF and $2.5 \mathrm{~g} / \mathrm{mL}$ of JPBXF treatments increased the numbers of WBCs and RBCs at different degrees. Nevertheless, the number of PLTs showed a slight increase in GM-CSF group, but had few changes in CTX and JPBXF groups (Figure 3A). Meanwhile, ELISA assays showed that the secretion levels of GM-CSF, EPO, and TPO were significantly reduced by $35.05 \pm$ $7.99 \%, 89.84 \pm 2.48 \%$, and $49.48 \pm 4.79 \%$ respectively in CTX group compared with the control group. However, JPBXF treatments abrogated the suppression effect of CTX and markedly increased the secretion levels of GM-CSF, EPO, and TPO at different degrees in plasma. Compared with CTX group,

TABLE 1 | Compounds of aqueous extract from Jian-pi-bu-xue-formula (JPBXF) were identified.

\begin{tabular}{|c|c|c|c|c|c|c|}
\hline No. & $T_{R}(\min )$ & $\mathrm{m} / \mathbf{z}$ & Formula & Identification & Therotical m/z & Error (ppm) \\
\hline 1 & 5.81 & 165.0545 & $\mathrm{C}_{9} \mathrm{H}_{10} \mathrm{O}_{3}$ & paeonol & 165.0557 & 7.3 \\
\hline 2 & 23.21 & 549.1582 & $\mathrm{C}_{26} \mathrm{H}_{30} \mathrm{O}_{13}{ }^{\mathrm{a}}$ & liquiritin apioside & 549.1614 & 5.8 \\
\hline 3 & 23.34 & 417.1170 & $\mathrm{C}_{21} \mathrm{H}_{22} \mathrm{O}_{9}^{\mathrm{b}}$ & liquiritin & 417.1191 & 5.0 \\
\hline 4 & 24.74 & 623.1952 & $\mathrm{C}_{29} \mathrm{H}_{36} \mathrm{O}_{15}{ }^{\mathrm{c}}$ & acteoside & 623.1981 & 4.6 \\
\hline 5 & 26.07 & 579.1672 & $\mathrm{C}_{27} \mathrm{H}_{32} \mathrm{O}_{14}$ & naringin & 579.1719 & 8.1 \\
\hline 6 & 26.64 & 623.1963 & $\mathrm{C}_{29} \mathrm{H}_{36} \mathrm{O}_{15}{ }^{\mathrm{c}}$ & isoacteoside & 623.1980 & 2.7 \\
\hline 7 & 28.74 & 609.1791 & $\mathrm{C}_{28} \mathrm{H}_{34} \mathrm{O}_{15}$ & hesperidin & 609.1825 & 5.6 \\
\hline 8 & 32.37 & 549.1589 & $\mathrm{C}_{26} \mathrm{H}_{30} \mathrm{O}_{13}{ }^{\mathrm{a}}$ & isoliquiritin apioside & 549.1614 & 4.5 \\
\hline 9 & 33.67 & 417.1176 & $\mathrm{C}_{21} \mathrm{H}_{22} \mathrm{O}_{9}^{\mathrm{b}}$ & isoliquiritin & 417.1191 & 3.6 \\
\hline 10 & 36.61 & 983.4429 & $\mathrm{C}_{48} \mathrm{H}_{72} \mathrm{O}_{21}$ & licoricesaponine A3 & 983.4493 & 6.5 \\
\hline 11 & 36.76 & 255.0659 & $\mathrm{C}_{15} \mathrm{H}_{12} \mathrm{O}_{4}$ & liquiritigenin & 255.0663 & 1.6 \\
\hline 12 & 39.38 & 283.0604 & $\mathrm{C}_{16} \mathrm{H}_{12} \mathrm{O}_{5}$ & calycosin & 283.0612 & 2.8 \\
\hline 13 & 39.91 & 837.3864 & $\mathrm{C}_{42} \mathrm{H}_{62} \mathrm{O}_{17}$ & licoricesaponine G2 & 837.3914 & 6.0 \\
\hline 14 & 46.48 & 821.3915 & $\mathrm{C}_{42} \mathrm{H}_{62} \mathrm{O}_{16}$ & glycyrrhizic acid & 821.3965 & 6.1 \\
\hline 15 & 58.61 & 267.0655 & $\mathrm{C}_{16} \mathrm{H}_{12} \mathrm{O}_{4}$ & formononetin & 267.0663 & 3.0 \\
\hline 16 & 65.18 & 367.1188 & $\mathrm{C}_{21} \mathrm{H}_{20} \mathrm{O}_{6}$ & glycycoumarin & 367.1187 & 0.3 \\
\hline 17 & 65.26 & 265.1476 & $\mathrm{C}_{15} \mathrm{H}_{22} \mathrm{O}_{4}$ & 4-gingerol & 265.1445 & 7.9 \\
\hline 18 & 67.02 & 351.0878 & $\mathrm{C}_{20} \mathrm{H}_{16} \mathrm{O}_{6}$ & licoisoflavone B & 351.0874 & 1.1 \\
\hline
\end{tabular}

\footnotetext{
${ }^{a, b, c}$ Three pairs of flavonoids isomers.
} 


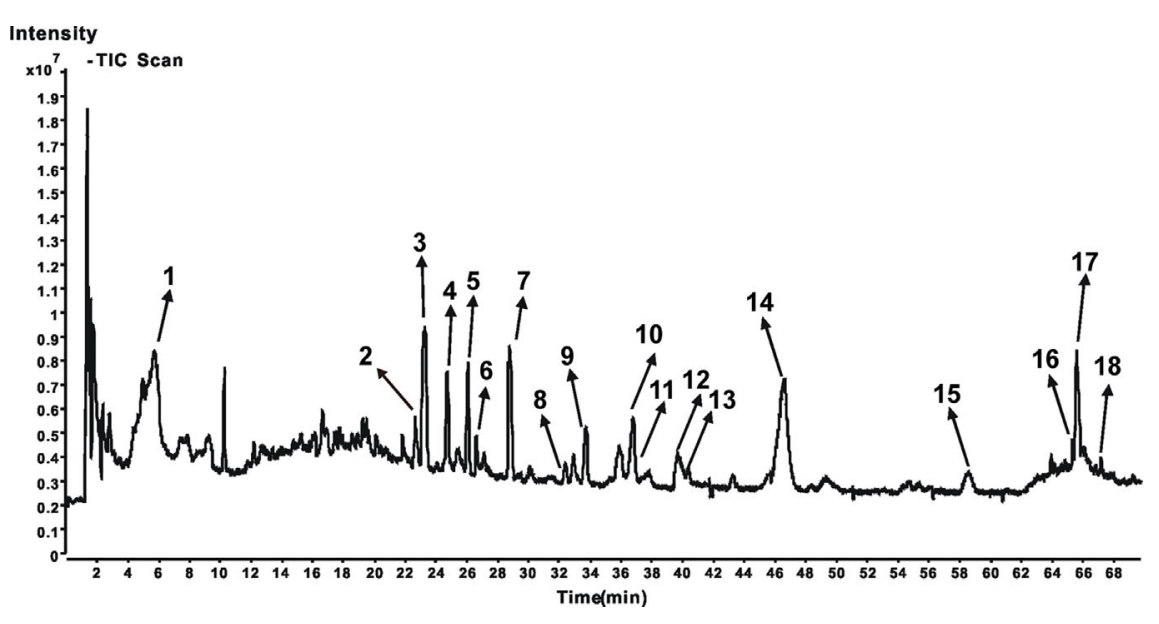

FIGURE 1 | Chromatograms of UHPLC/MS/TOF of JPBXF extract.

$2.5 \mathrm{~g} / \mathrm{mL}$ of JPBXF treatments increased the levels of GM-CSF, EPO and TPO by 2.94-, 69.84-, and 4.21-fold in plasma (Figure 3B).

\section{JPBXF Reduced CTX-Induced Immunosuppression}

According to the white blood cell analysis results, we found that JPBXF treatments might raise the immunity of mice suppressed by CTX. Among these white blood cells, T cell play important role in immunity. To further confirm the effect of JPBXF on T cells, we detected the expressions of T-cell surface markers, such as CD3, CD4, and CD8 $\alpha$ in PBMCs. The results demonstrated that compared with control group, CTX treatment significantly decreased the expressions of CD3, CD 4 , and CD $8 \alpha$ by $44.03 \pm$ $2.96 \%, 14.63 \pm 4.14 \%$, and $46.70 \pm 3.10 \%$, respectively. However, CTX-induced expression decrease of CD3, CD4 and CD8 $\alpha$ were recovered by JPBXF treatments (Figure 4). In detail, $2.5 \mathrm{~g} / \mathrm{mL}$ of JPBXF treatments increased CD3, CD4, and CD8a expression levels by $31.33 \pm 5.59 \%, 28.62 \pm 3.44 \%$, and $18.13 \pm 2.24 \%$, respectively. Meanwhile, $0.62 \mathrm{~g} / \mathrm{mL}$ of JPBXF treatments increased the expressions of CD3, CD4, and CD8a by $37.99 \pm$ $5.07 \%$, $34.06 \pm 3.09 \%$, and $45.16 \pm 3.29 \%$, respectively.

Thymus and spleen are two main immune organs that play important roles in cellular and humoral immunity. To assess the effects of JPBXF on cellular and humoral immunity in mice, size comparison and $\mathrm{H} \& \mathrm{E}$ staining of thymus and spleen were further conducted. The pictures of thymus directly showed that CTX treatment induced a size reduce of thymus compared with that of control, however, JPBXF treatments increased the size of thymus. Compared with the control group, thymus index (ratios of thymus to body-weight) in CTX group decreased markedly. Meanwhile, thymus index in GM-CSF group and high-dose JPBXF group were significantly increased compared with CTX group; however, thymus index in the low-dose JPBXF group had no significant change (Figure 5A). H\&E staining results showed that the tissue structures of thymus, including cortex, medulla, and thymic corpuscle, were clear observed in control group, and destroyed in CTX group. However, the structure damages of thymus by CTX were significantly recovered by JPBXF treatments (Figure 5B). The same as thymus, the size of spleen was reduced by CTX and increased by JPBXF. Spleen index (ratios of spleen to body-weight) in CTX group was decreased markedly compared with that of control group, meanwhile, increased in GM-CSF and JPBXF groups compared with CTX group (Figure 6A). In addition, white pulp atrophy, hemorrhage and necrosis were observed in spleen after CTX treatment. However, these damages were reduced in GM-CSF and JPBXF groups, especially in $2.5 \mathrm{~g} / \mathrm{mL}$ JPBXF group (Figure 6).

\section{JPBXF Alleviated CTX-Induced Myelosuppression Through Activating NRF2/HO1/NQO1 Signaling Pathway}

NRF2, a pivotal transcription factor, is involved in regulating redox homeostasis, drug metabolism, responses to oxidative and electrophilic stress and so on (Hayes and DinkovaKostova, 2014). NRF2 deficiency deteriorated CTX-induced myelosuppression, while activation of NRF2 mitigated CTXinduced myelosuppression (Que et al., 2016). Herein, to further determine the underlying mechanism of JPBXF in alleviating CTXinduced myelosuppression, NRF2 expressions in thymus or spleen were evaluated. Our results showed that the expressions of NRF2 were significantly decreased by CTX both in thymus and spleen compared with control group, meanwhile, notably increased by GM-CSF and JPBXF treatments compared with CTX group, suggesting that JPBXF could mitigate CTX-induced myelosuppression through activating of NRF2 (Figure 7A). The up-regulation of $\mathrm{HO} 1$ and NQO1 could resist to CTX-induced bone marrow suppression, oxidative stress, inflammation and apoptosis (Chen et al., 2014; HAS et al., 2019), and we found that JPBXF reversed CTX-induced $\mathrm{HO} 1$ and NQO1 suppressions both in mRNA and protein levels (Figures 7B-D), indicating that JPBXF alleviated CTX-induced myelosuppression through activating NRF2/HO1/NQO1 signaling pathway. 

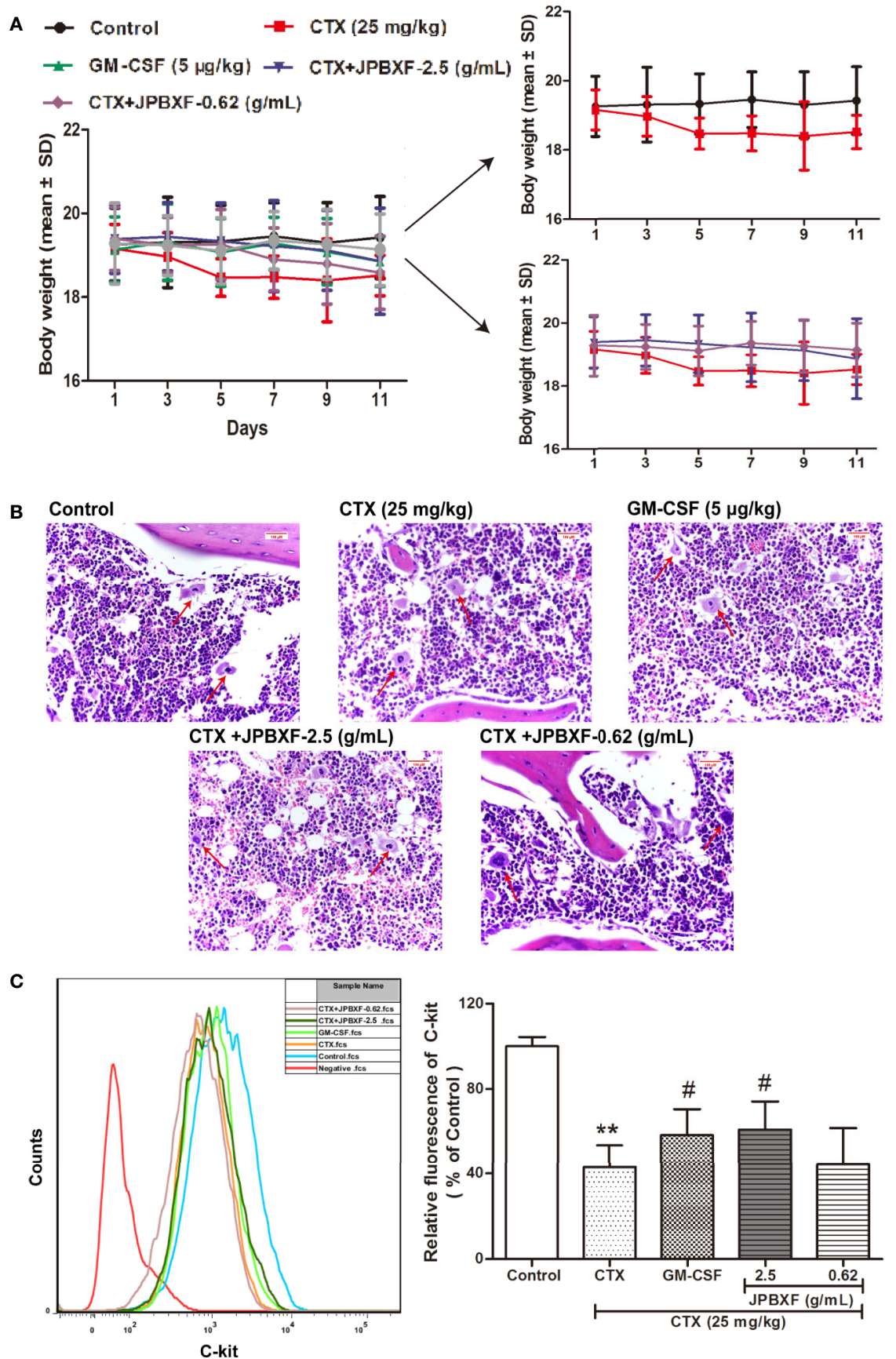

FIGURE 2 | Effects of JPBXF on myelosuppression in CTX-treated mice. (A) The body weight curves of CTX-treated mice after JPBXF treatments. (B) Histopathological changes in bone marrow tissues (stained by H\&E, $\times 400$ ). (C) Expression of C-kit in bone marrow cells. Data shown are means \pm SD from day 13. ${ }^{\star \star} P<0.01$ vs. Control, ${ }^{\#} P<0.05$ vs. CTX.

\section{Target Prediction of Eighteen Compounds on Alleviating Myelosuppression by Network Pharmacology}

We queried 3,519 genes related to myelosuppression using GeneCards database, 482 predicted target genes of compounds using BATMAN-TCM database and 582 predicted target genes of compounds using Swiss Target Prediction database. 87 of the potential targets yielded from these three database were selected for GO and KEGG analyses (Figure 8A). P $<0.05$ was set as threshold criteria to identify the functional gene ontology and pathway, and Top 20 ontology and pathway were showed in Figures 8B, C. GO enrichment analysis indicated that the 87 
A
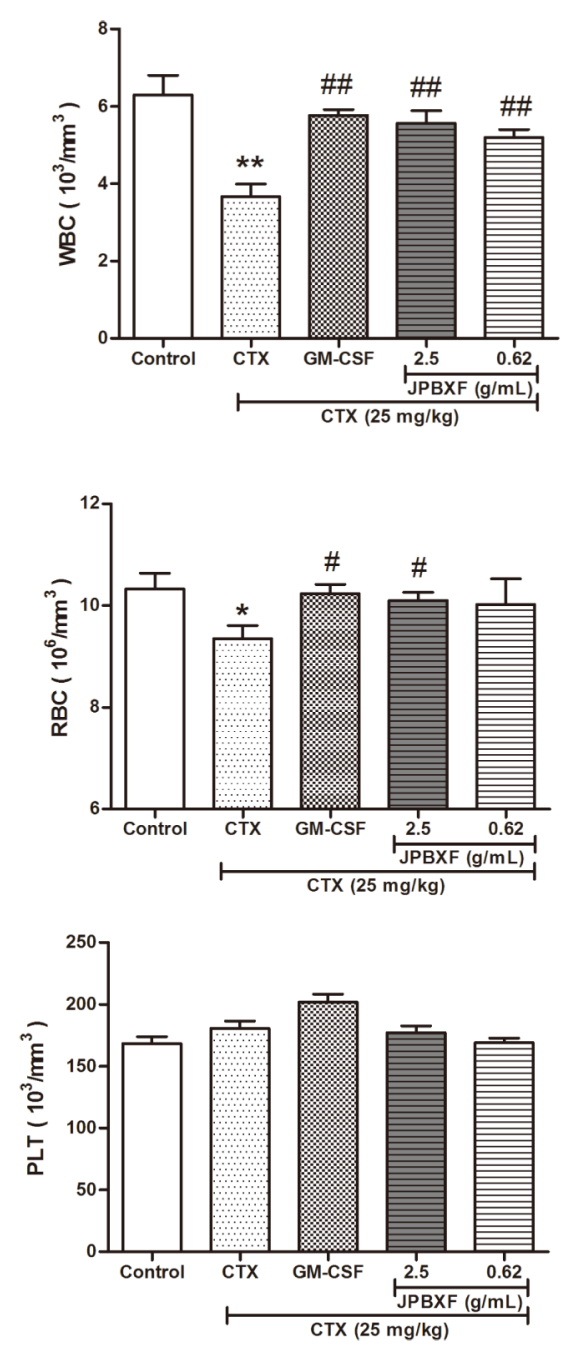

B
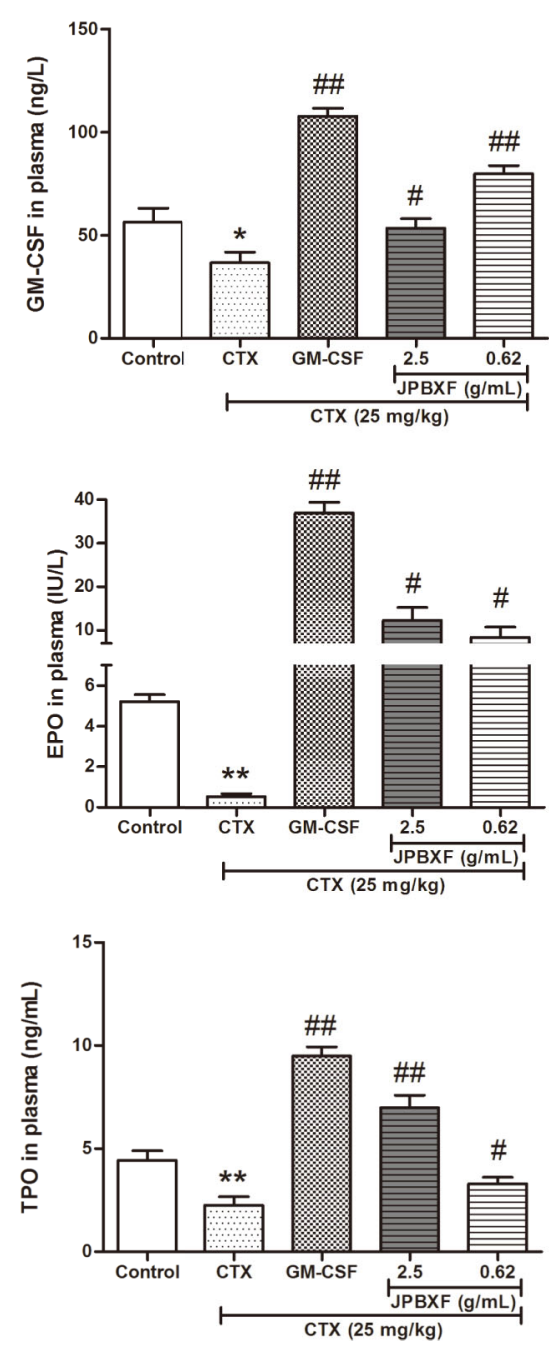

FIGURE 3 | Effects of JPBXF on blood system in CTX-treated mice. (A) The numbers of white blood cells, red blood cells and platelets in CTX-treated mice after JPBXF treatments. (B) The secretion levels of GM-CSF, EPO, and TPO in plasma in CTX-treated mice after JPBXF treatments. Data shown are means \pm SD from day 13. ${ }^{\star} P<0.05,{ }^{\star \star} P<0.01$ vs. Control, ${ }^{\#} P<0.05,{ }^{\# \#} P<0.01$ vs. CTX.

potential targets were primarily associated with the "response to organic substance," "response to chemical," "multicellular organismal process," "regulation of multicellular organismal process," and "regulation of biological quality" terms. KEGG enrichment analysis revealed that the 87 potential targets were significantly enriched in the "Neuroactive ligand-receptor interaction," "Pathways in cancer," "Calcium signaling pathway" "cAMP signaling pathway" and "Vascular smooth muscle contraction" terms. The PPI network identified 14 key genes for the compounds, including AGTR2, PTGS2, MTOR, DRD2, EP300, OPRM1, AGTR1, DRD3, OPRD1, OPRK1, PPARG, PTGER3, RXRA, and TNF (Figure 8D). The three-level network consisted of 125 nodes (18 compounds, 87 genes and 20 pathways) and 552 edges. Among the 18 compounds, naringin showed the broadest effect on the target genes (38 genes). Liquiritin apioside, liquiritigenin, licoricesaponine G2, and isoliquiritin also affected 36, 35, 33, and 33 genes, respectively. Among the 87 genes, PRKCA, PRKCB and IMPDH1 were influenced by 16,13 , and 12 compounds, respectively. OPRD1, PPP2CA, ESR1, OPRM1, OPRK1, and ALOX5 were affected by 11 compounds. CNR2 was affected by 10 compounds. IMPDH2 was influenced by 9 compounds. DRD2, NR3C1 and TOP2A were affected by 8 compounds. While three targets were influenced by 7 compounds, one target was influenced by 6 compounds, ten targets were affected by 5 compounds, nine targets were affected by 4 compounds, twenty three targets were affected by 3 compounds, and the rest twenty seven were affected by only one compound. 
A

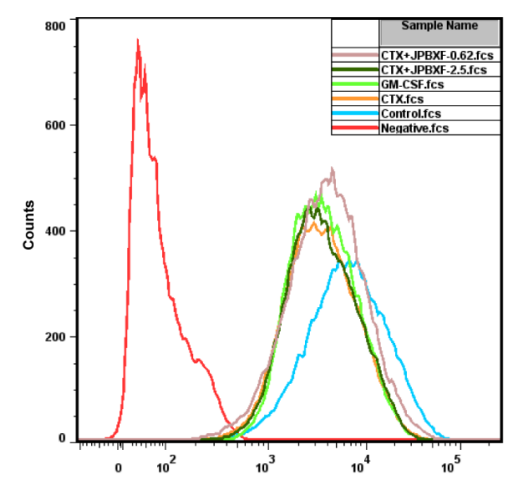

B

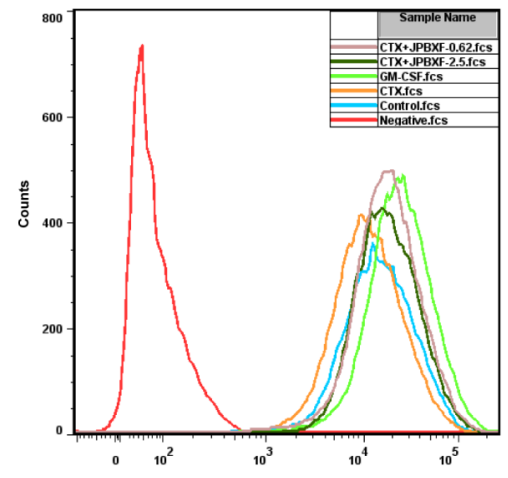

C

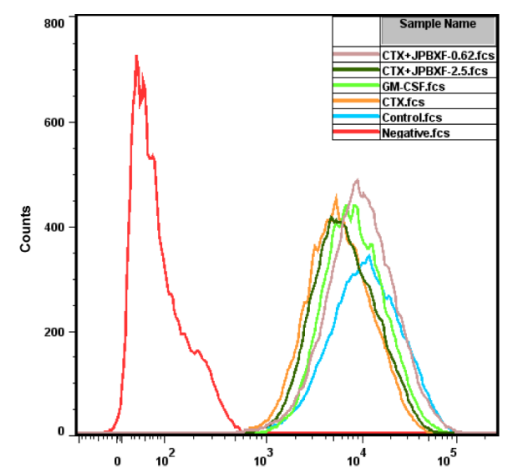

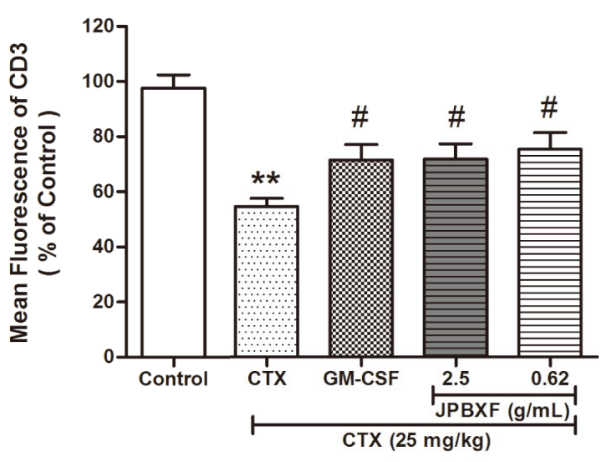
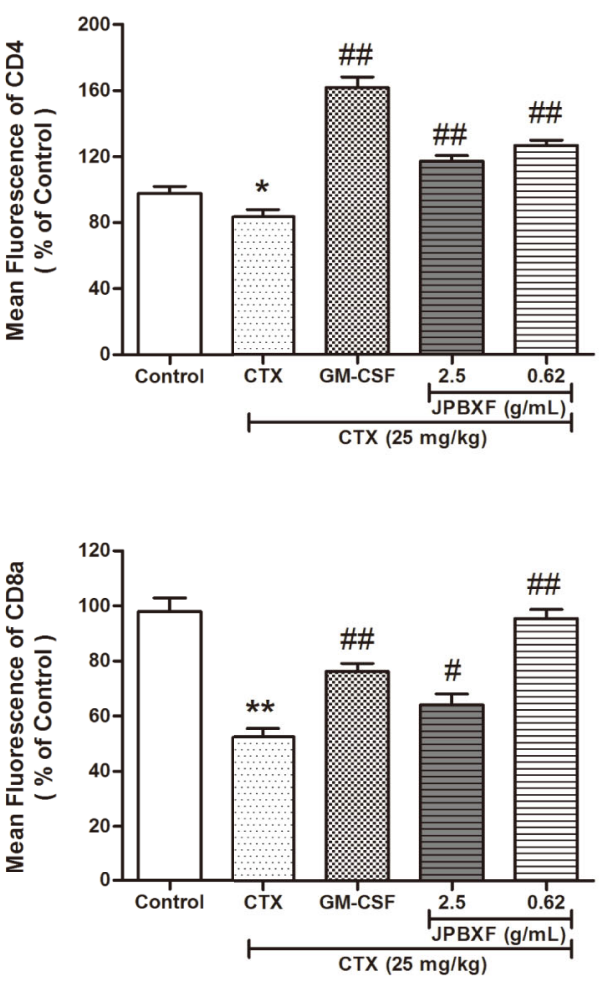

FIGURE 4 | Effects of JPBXF on the PBMCs in CTX-treated mice. The expression levels of CD3 (A), CD4 (B), and CD8 $\alpha$ (C) in peripheral blood mononuclear cells (PBMCs) of CTX-treated mice after JPBXF treatments. Data shown are means \pm SD from day $13 .{ }^{*} P<0.05,{ }^{\star *} P<0.01$ vs. Control, ${ }^{\sharp} P<0.05$, ${ }^{\# \#} P<0.01$ vs. CTX.

\section{DISCUSSION}

TCMs are widely used in Asian countries, especially China, Japan and Korea. With the development of medicine, Chinese medicine is gradually accepted by western countries (Dobos and Tao, 2011). Given the ability of alleviating chemotherapyinduced side effects, TCMs complementary treatment is widely adopted for patients suffering from cancers (Woo et al., 2016). To be an empirical TCM formula, JPBXF has been used to ease chemotherapy-induced weakness and fatigue for cancer patients in clinic in China for decades. However, the phytochemical characterization, detail therapeutic evaluation and underline mechanism of JPBXF remain unclear.

Our study directly provides evidence that JPBXF treatments could alleviate CTX-induced myelosuppression in C57BL/6 mice as following aspects: (i) JPBXF treatments increased the number of HSCs; (ii) JPBXF treatments recovered CTX-induced blood cells reduction; (iii) JPBXF treatments reduced CTXinduced immunosuppression. Cancer patients who receive CTX-chemotherapy, often suffered side effects including myelosuppression, weight loss, asthenia, and so on (Strati et al., 2013; Que et al., 2016). In our study, JPBXF treatments 
A
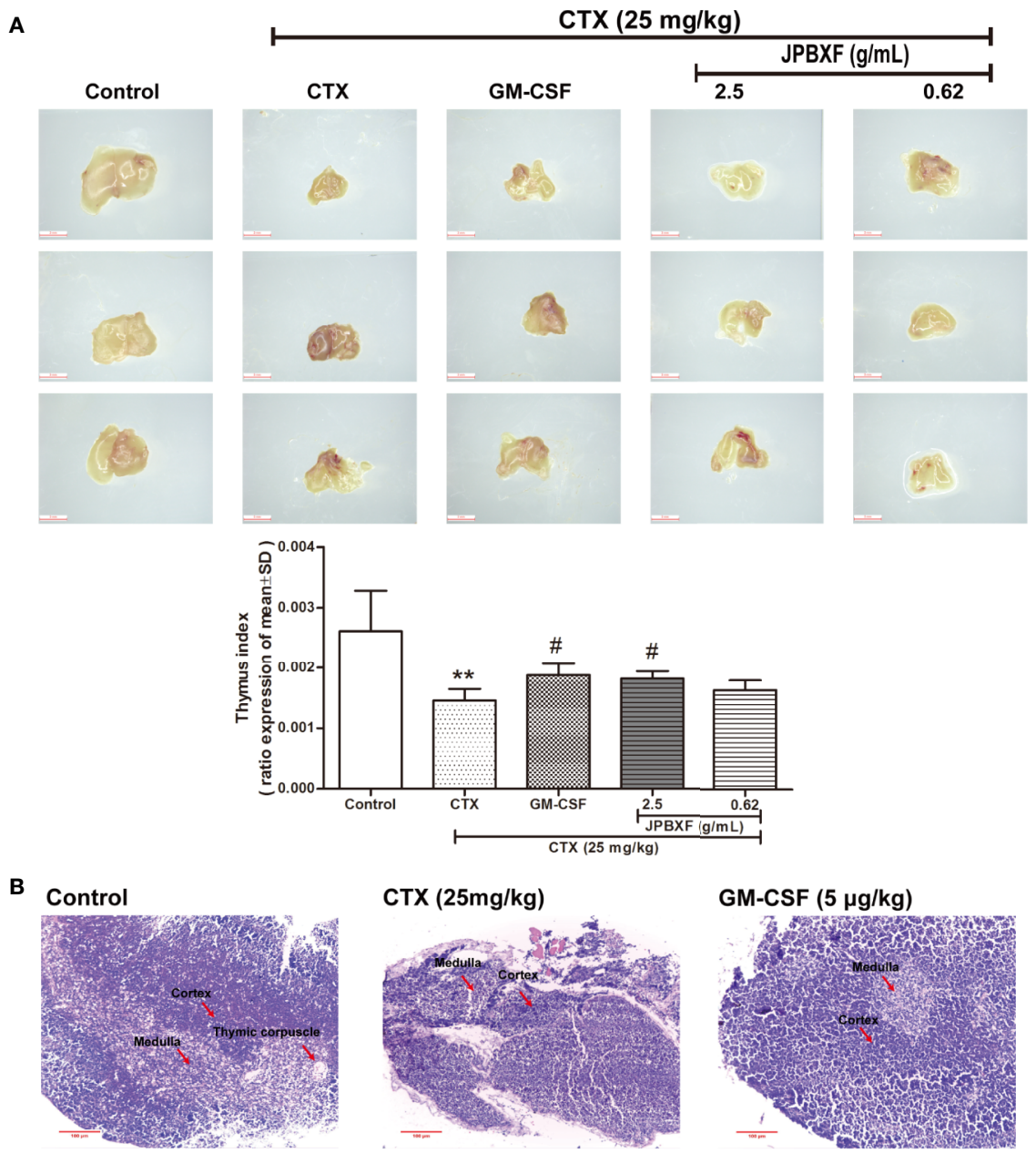

GM-CSF $(5 \mu \mathrm{g} / \mathrm{kg})$

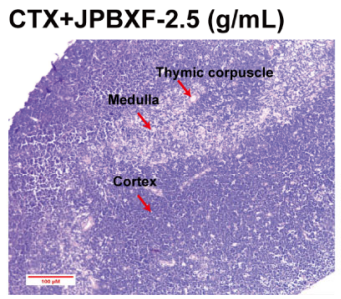

CTX+JPBXF-0.62 (g/mL)

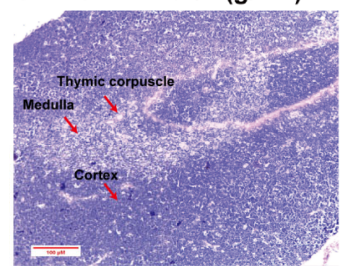

FIGURE 5 | Effects of JPBXF on the thymus in CTX-treated mice. (A) Sizes and index of thymus in mice (scale $=3$ mm). (B) Histopathological changes in thymus tissues (stained by H\&E, $\times 200$ ). Data shown are means \pm SD from day 13 . ${ }^{\star *} P<0.01$ vs. Control, ${ }^{\#} P<0.05$ vs. CTX.

could increase the number of HSCs and the expression of HSC marker C-kit (Figure 2), illustrating that JPBXF could abrogate the myelosuppression induced by CTX. On the other hand, CTX treatment could significantly reduce the number of WBCs and RBCs and the secretion levels of GM-CSF, EPO and TPO in mice, which consistent with the previous reports (Botnick et al., 1981; Jantunen et al., 2003). Expectedly, JPBXF treatments increased the number of RBCs and WBCs, and the secretion levels of EPO and GM-CSF at different degrees (Figure 3). Except for increasing blood function, JPBXF also showed great ability on reducing CTX-induced immunosuppression in C57BL/6 mice. It is reported that CTX treatment could induce immunosuppression and inhibit the generation and function of T cells (Traverso et al., 2012; Salva et al., 2014). CD3, CD4, and CD8a are the most abundant immunity $\mathrm{T}$ cell in the PBMCs (Sedgmen et al., 2013). We found that the expressions of CD3, CD4, and CD8a in PBMCs were decreased by CTX, while JPBXF abrogated the decrease expressions of CD3, CD4 and CD8a (Figure 4). Furthermore, thymus and spleen are two main immune organs that play important roles in cellular and humoral immunity. Thymus and spleen functions can be inhibited by CTX treatment (Zhao et al., 2009). In the present study, we found that thymic atrophy and splenatrophy were obvious after CTX treatment. To the contrary, thymus and 
A

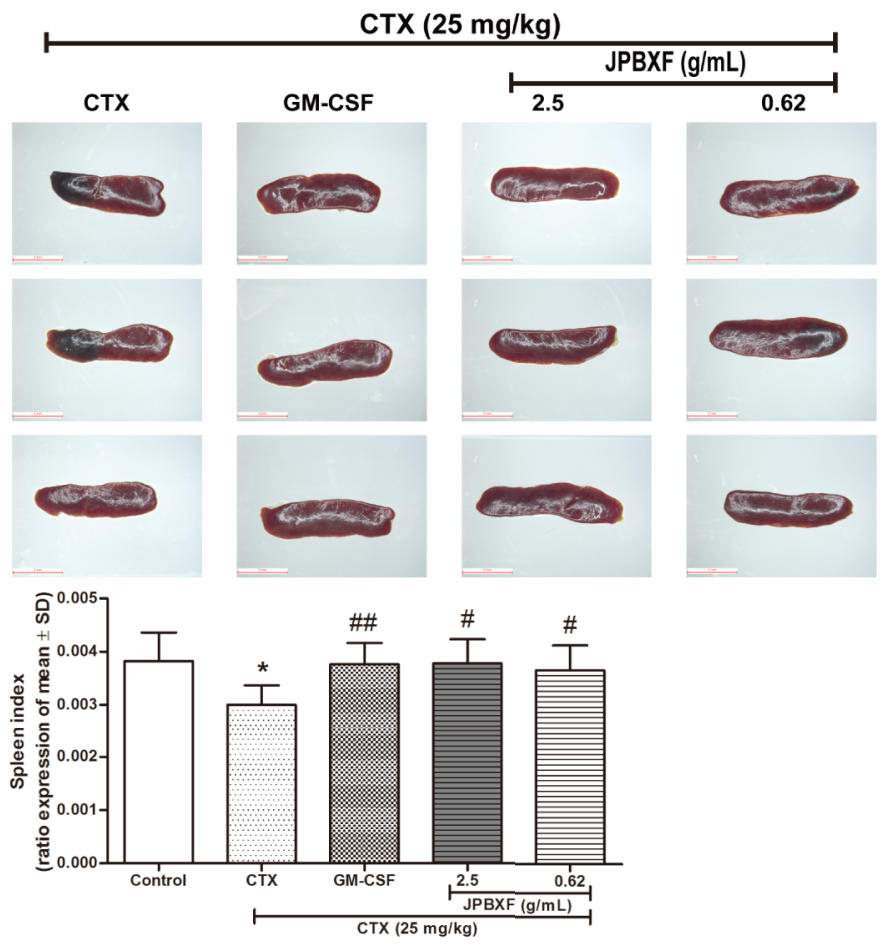

B Control

CTX $(25 \mathrm{mg} / \mathrm{kg})$

GM-CSF (5 $\mu \mathrm{g} / \mathrm{kg})$
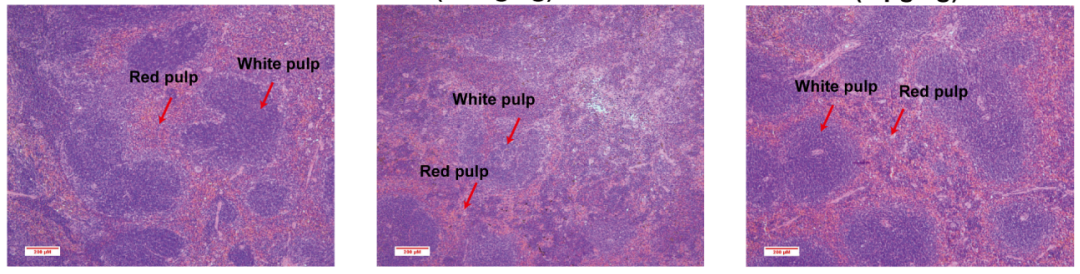

CTX+JPBXF-2.5 (g/mL)

CTX+JPBXF-0.62 (g/mL)
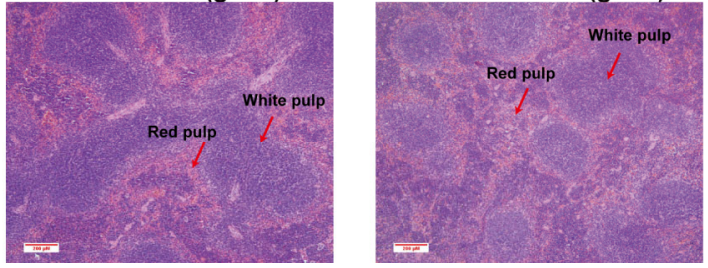

FIGURE 6 | Effects of JPBXF on the spleen in CTX-treated mice. (A) Sizes and index of spleens in mice (scale=5 mm). (B) Histopathological changes in spleens tissues (stained by H\&E, $\times 100$ ). Data shown are means \pm SD from day $13 .{ }^{*} P<0.05$ vs. Control, ${ }^{\#} P<0.05,{ }^{\# \#} P<0.01$ vs. CTX.

spleen index increased after JPBXF treatments (Figures 5A and 6A). In addition, CTX-induced structure damages, including cortex, medulla and thymic corpuscle in thymus and white pulp atrophy, hemorrhage and necrosis in spleen, were recovered by JPBXF treatments (Figures 5B and 6B).

Our data also revealed that JPBXF alleviated CTX-induced myelosuppression through activating NRF2/HO1/NQO1 signaling pathway. It is reported that NRF2 deficiency deteriorated CTXinduced myelosuppression, while activation of NRF2 mitigates CTX -induced myelosuppression (Que et al., 2016). The up-regulation of
HO1 or NQO1 could resist to CTX-induced bone marrow suppression, oxidative stress, inflammation and apoptosis (Chen et al., 2014; HAS et al., 2019). We observed that JPBXF treatment notably reversed CTX-induced NRF2 suppressions both in thymus and spleen, subsequently, recovered the expression of $\mathrm{HO} 1$ and NQO1 both in mRNA and protein levels (Figure 7), indicating that JPBXF alleviated CTX-induced myelosuppression through activating NRF2/HO1/NQO1 signaling pathway.

In our study, JPBXF aqueous extract showed great ability in alleviating CTX-induced myelosuppression in C57BL/6 mice. 
A

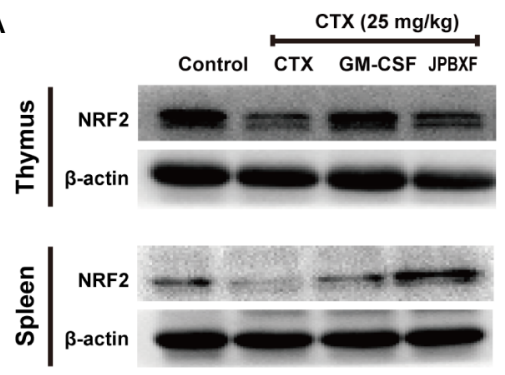

B

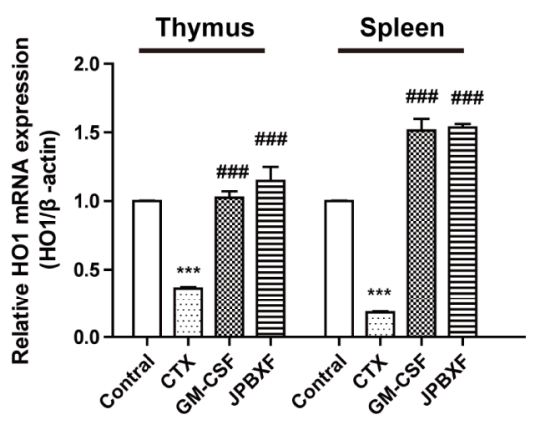

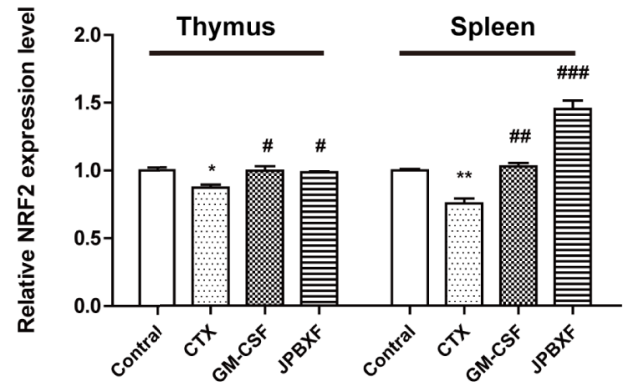

C

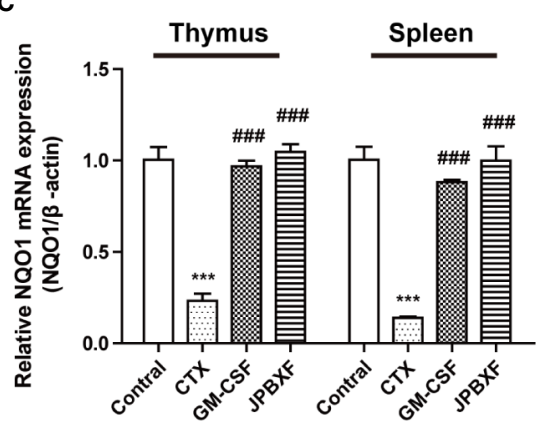

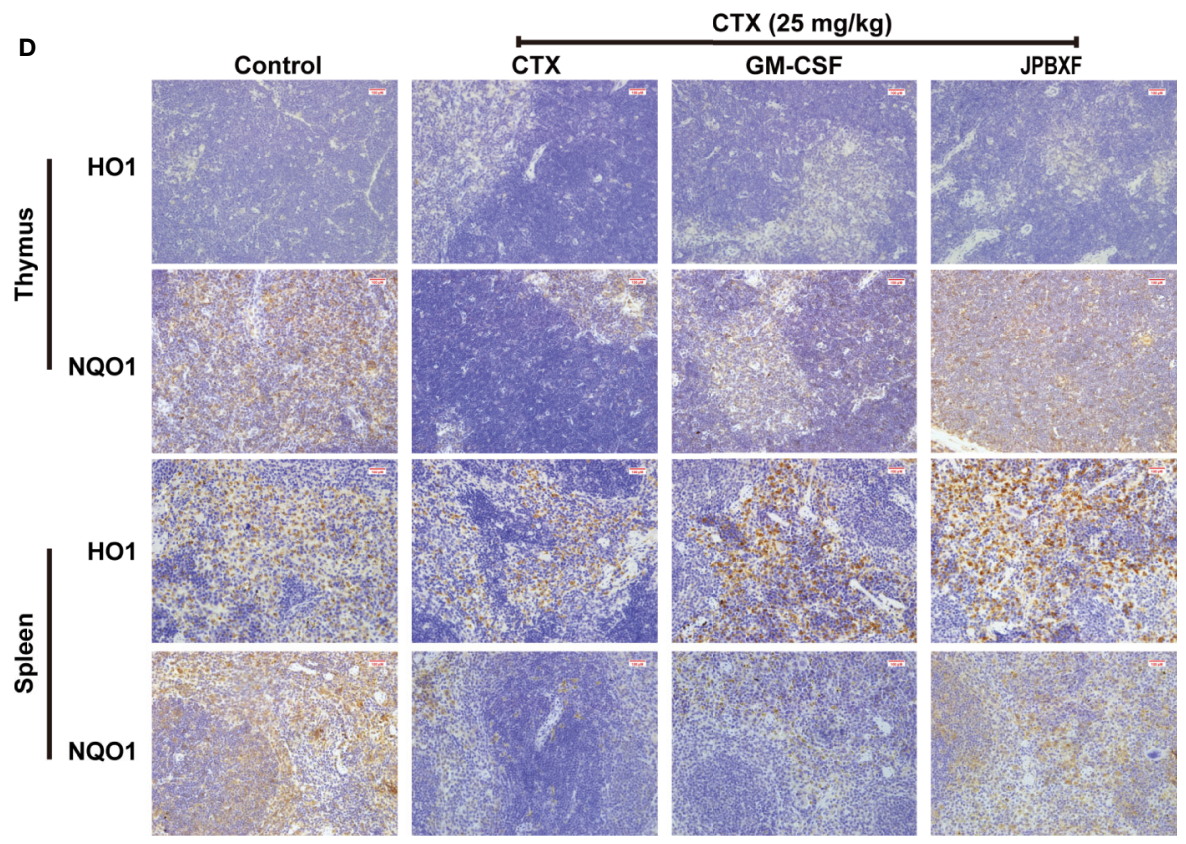

FIGURE 7 | JPBXF activated NRF2/HO1/NQO1 pathway. (A) Protein expressions of NRF2 in thymus or spleen tissues after treatments as design. mRNA expression levels of $\mathrm{HO1}$ (B) and NQO1 (C) were detected using real-time PCR analysis. (D) Images of $\mathrm{HO} 1$ and NQO1 protein expressions in thymus or spleen tissues were presented by immunohistochemistry. Data shown are means \pm SD from day $13 .{ }^{\star} P<0.05,{ }^{\star *} P<0.01,{ }^{\star \star \star} P<0.001$ vs. Control, ${ }^{\#} P<0.05,{ }^{\# \#} P<0.01$, \#\#\# $P<0.001$ vs. CTX.

However, the main chemical constituents in JPBXF aqueous extract and the underline mechanisms of the main compounds on alleviating CTX-induced myelosuppression are still unclear. Herein, we identified main chemical constituents of JPBXF aqueous extract using UHPLC/MS/TOF and confirmed that the main constituents in JPBXF extracts are paeonol, liquiritin apioside, liquiritin, acteoside, naringin, isoacteoside, hesperidin, isoliquiritin apioside, isoliquiritin, licoricesaponine $\mathrm{A} 3$, liquiritigenin, calycosin, licoricesaponine G2, glycyrrhizic acid, formononetin, glycycoumarin, 


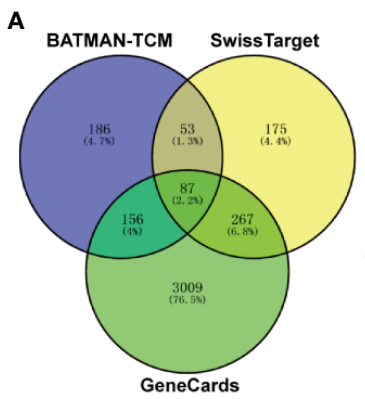

D

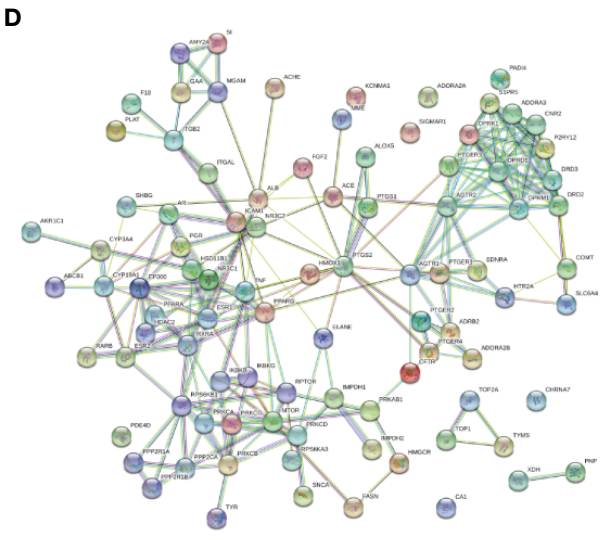

B

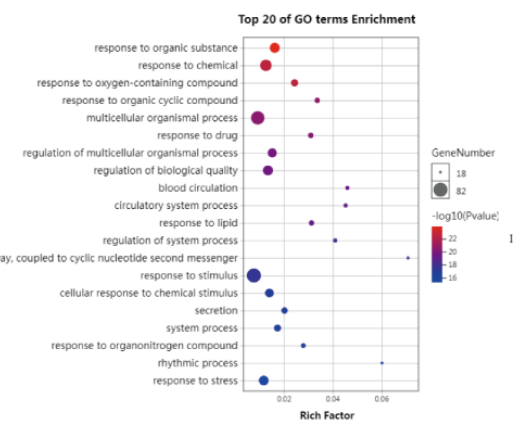

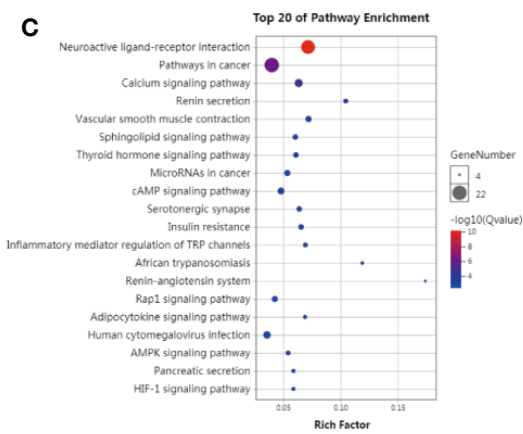

E

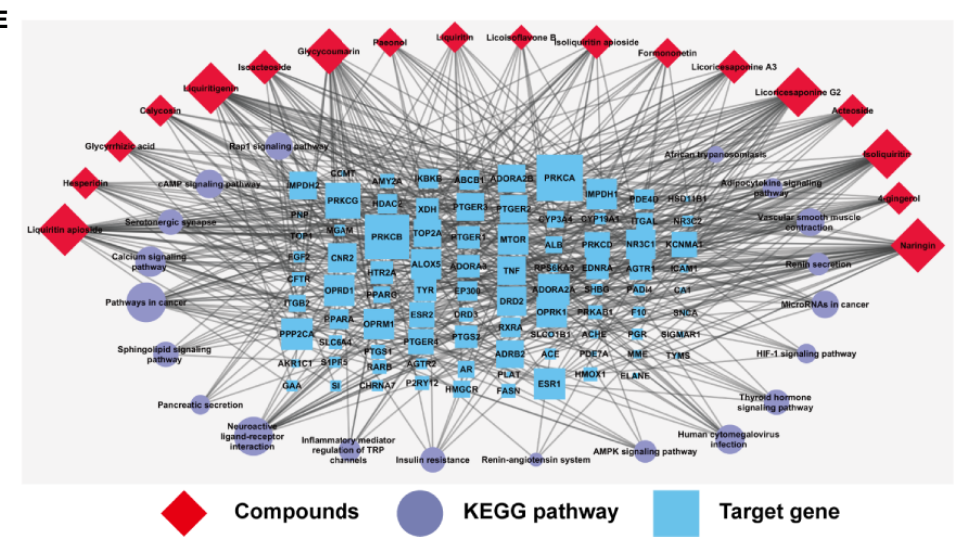

FIGURE 8 | Target prediction of eighteen compounds on alleviating myelosuppression. (A) The 87 potential target genes of eighteen compounds on myelosuppression were selected using Venny. (B) Gene ontology (GO) enrichment analysis of 87 selected genes performed by Database for Annotation, Visualization and Integrated Discovery (DAVID) and visualized by Omicshare. (C) KEGG enrichment analysis of 87 selected genes performed by DAVID and visualized by Omicshare. (D) The protein-protein interaction (PPI) network of 87 selected genes constructed by the STRING database. (E) The compound-target-pathway network was constructed and visualized by Cytoscape.

4-gingerol, and licoisoflavone B (Figure 1 and Table 1). We also try to explain the underline mechanism by which 18 compounds on alleviating CTX-induced myelosuppression using bioinformatics analysis and found that 87 potential targets were primarily associated with the "response to organic substance," "response to chemical," "multicellular organismal process," "regulation of multicellular organismal process," and "regulation of biological quality" terms by targeting multi-protein network, such as "Neuroactive ligandreceptor interaction," "Pathways in cancer," "Calcium signaling pathway" "cAMP signaling pathway," and "Vascular smooth muscle contraction". Combining with the PPI network and the three-level network results, 24 identified genes, including AGTR2, PTGS2, MTOR, DRD2, EP300, OPRM1, AGTR1, DRD3, OPRD1, OPRK1, PPARG, PTGER3, RXRA, TNF, PRKCA, PRKCB, IMPDH1, PPP2CA, ESR1, ALOX5, CNR2, IMPDH2, NR3C1, and TOP2A (Figure 8), might be the key targets of 18 compounds in alleviating CTX-induced myelosuppression. To confirm these prediction results, more researches will be conducted in the future.

In conclusion, JPBXF can greatly reverse CTX-induced myelosuppression in $\mathrm{C} 57 \mathrm{BL} / 6$ mice, especially in improving the blood and immune function through activating NRF2/ HO1/NQO1 signaling pathway, which provides a reliable reference for JPBXF application in clinical. By recognizing 18 compounds in JPBXF aqueous extract and predicting the underline mechanism, our study would provide theoretical guidance for further research of JPBXF.

\section{DATA AVAILABILITY STATEMENT}

The raw data supporting the conclusions of this article will be made available by the authors, without undue reservation, to any qualified researcher.

\section{ETHICS STATEMENT}

The animal study was reviewed and approved by International Institute for Translational Chinese Medicine Animal Care and Use Committee, Guangzhou University of Chinese Medicine. 


\section{AUTHOR CONTRIBUTIONS}

LL and XL designed the research. QH, LF, HL, and LZ performed the study. QH and LF analyzed the data and wrote the manuscript. ZL and QF revised the manuscript. XQ and YW provided technical support. All authors contributed to the article and approved the submitted version.

\section{REFERENCES}

Botnick, L. E., Hannon, E. C., Vigneulle, R., and Hellman, S. (1981). Differential effects of cytotoxic agents on hematopoietic progenitors. Cancer Res. 41 (6), 2338-2342.

Bray, F., Ferlay, J., Soerjomataram, I., Siegel, R. L., Torre, L. A., and Jemal, A. (2018). Global cancer statistics 2018: GLOBOCAN estimates of incidence and mortality worldwide for 36 cancers in 185 countries. CA Cancer J. Clin. 68 (6), 394-424. doi: 10.3322/caac.21492

Carey, P. J. (2003). Drug-induced myelosuppression : diagnosis and management. Drug Saf. Int. J. Med. Toxicol. Drug Exp. 26 (26), 691-706. doi: 10.2165/ 00002018-200326100-00003

Chen, S., Wang, J., Fang, Q., Gao, R., Shi, Q., Zhang, H., et al. (2014). Upregulated heme oxygenase-1 expression of mouse mesenchymal stem cells resists to chemotherapy-induced bone marrow suppression. Chin. Med. J. (Engl) 127 (7), 1310-1316. doi: 10.3760/cma.j.issn.0366-6999.20133214

Cohen, I., Tagliaferri, M., and Tripathy, D. (2002). Traditional Chinese medicine in the treatment of breast cancer. Semin. Oncol. 29 (6), 563-574. doi: 10.1053/ sonc. 2002.50005

Dobos, G., and Tao, I. (2011). The model of Western integrative medicine: the role of Chinese medicine. Chin. J. Integr. Med. 17 (1), 11-20. doi: 10.1007/s11655011-0601-x

Du, Z., Zhang, S., Lin, Y., Zhou, L., Wang, Y., Yan, G., et al. (2019). Momordicoside G Regulates Macrophage Phenotypes to Stimulate Efficient Repair of Lung Injury and Prevent Urethane-Induced Lung Carcinoma Lesions. Front. Pharmacol. 10, 321 doi: 10.3389/fphar.2019.00321

Feng, L., and Liu, X. (2015). Effects of Jianpi Buxue Decoction on EPO, TPO, and GM-CSF in Lewis Lung Cancer Mice Treated with Chemotherapy. Trad. Chin. Drug Res. Clin. Pharmacol. 26, 431-434. doi: 10.3969/j.issn.10039783.2015.04.002

Feng, L., Huang, Q., Huang, Z., Li, H., Qi, X., Wang, Y., et al. (2016). Optimized Animal Model of Cyclophosphamide-induced Bone Marrow Suppression. Basic Clin. Pharmacol. Toxicol. 119 (5), 428-435. doi: 10.1111/bcpt.12600

Gong, A. G., Lau, K. M., Zhang, L. M., Lin, H. Q., Dong, T. T., and Tsim, K. W. (2016). Danggui Buxue Tang, Chinese Herbal Decoction Containing Astragali Radix and Angelicae Sinensis Radix, Induces Production of Nitric Oxide in Endothelial Cells: Signaling Mediated by Phosphorylation of Endothelial Nitric Oxide Synthase. Planta Med. 82 (5), 418-423. doi: 10.1055/s-0035-1558332

Gutschalk, C. M., Heroldmende, C. C., Fusenig, N. E., and Mueller, M. M. (2006). Granulocyte Colony-Stimulating Factor and Granulocyte-Macrophage Colony-Stimulating Factor Promote Malignant Growth of Cells from Head and Neck Squamous Cell Carcinomas In vivo. Cancer Res. 66 (16), 8026-8036. doi: 10.1158/0008-5472.CAN-06-0158

Hamsa, T. P., and Kuttan, G. (2010). Ipomoea obscura ameliorates cyclophosphamide-induced toxicity by modulating the immune system and levels of proinflammatory cytokine and GSH. Can. J. Physiol. Pharmacol. 88 (11), 1042-1053. doi: 10.1139/Y10-086

Handolias, D., Quinn, M., Foo, S., Mileshkin, L., Grant, P., Dutu, G., et al. (2016). Oral cyclophosphamide in recurrent ovarian cancer. Asia Pac. J. Clin. Oncol. 12 (1), e154-e160. doi: 10.1111/ajco.12074

HAS, A. L., Alotaibi, M. F., Bin-Jumah, M., Elgebaly, H., and Mahmoud, A. M. (2019). Olea europaea leaf extract up-regulates Nrf2/ARE/HO-1 signaling and attenuates cyclophosphamide-induced oxidative stress, inflammation and apoptosis in rat kidney. BioMed. Pharmacother. 111, 676-685. doi: 10.1016/ j.biopha.2018.12.112

\section{FUNDING}

This work was supported by the projects of the National Natural Science Foundation of China [81720108033, 81930114, and 81874367], the Natural Science Foundation of Guangdong Province [2018B030322011], and the Natural Science Funds for Distinguished Young Scholar of Guangdong Province [2017A030306033].

Hayes, J. D., and Dinkova-Kostova, A. T. (2014). The Nrf2 regulatory network provides an interface between redox and intermediary metabolism. Trends Biochem. Sci. 39 (4), 199-218. doi: 10.1016/j.tibs.2014.02.002

Hill, B. T., Rybicki, L., Smith, S., Dean, R., Kalaycio, M., Pohlman, B., et al. (2011). Treatment with hyperfractionated cyclophosphamide, vincristine, doxorubicin, and dexamethasone combined with cytarabine and methotrexate results in poor mobilization of peripheral blood stem cells in patients with mantle cell lymphoma. Leuk. Lymphoma 52 (6), 986-993. doi: 10.3109/10428194.2010.551154

Hubner, J., Prott, F. J., Micke, O., and Munstedt, K. (2015). [Complementary medicine and cancer]. Dtsch Med. Wochenschr 140 (10), 768-769. doi: 10.1055/ s-0041-102202

Jantunen, E., Putkonen, M., Nousiainen, T., Pelliniemi, T. T., Mahlamaki, E., and Remes, K. (2003). Low-dose or intermediate-dose cyclophosphamide plus granulocyte colony-stimulating factor for progenitor cell mobilisation in patients with multiple myeloma. Bone Marrow Transplant. 31 (5), 347-351. doi: 10.1038/sj.bmt.1703840

Konkimalla, V. B., and Efferth, T. (2008). Evidence-based Chinese medicine for cancer therapy. J. Ethnopharmacol. 116 (2), 207-210. doi: 10.1016/ j.jep.2007.12.009

Miller, R. C., and Steinbach, A. (2014). Growth factor use in medication-induced hematologic toxicity. J. Pharm. Pract. 27 (5), 453-460. doi: 10.1177/ 0897190014546113

Neboh, E. E., and Ufelle, S. A. (2015). Myeloprotective activity of crude methanolic leaf extract of Cassia occidentalis in cyclophosphamide-induced bone marrow suppression in Wistar rats. Adv. Biomed. Res. 4 (5), 5-5. doi: 10.4103/22779175.148285

Potti, A., Dressman, H. K., Bild, A., Riedel, R. F., Chan, G., Sayer, R., et al. (2011). Retraction: Genomic signatures to guide the use of chemotherapeutics. Nat. Med. 17 (1), 135. doi: 10.1038/nm0111-135

Que, L., He, L., Yu, C., Yin, W., Ma, L., Cao, B., et al. (2016). Activation of Nrf2ARE signaling mitigates cyclophosphamide-induced myelosuppression. Toxicol. Lett. 262, 17-26. doi: 10.1016/j.toxlet.2016.09.003

Salva, S., Marranzino, G., Villena, J., Aguero, G., and Alvarez, S. (2014). Probiotic Lactobacillus strains protect against myelosuppression and immunosuppression in cyclophosphamide-treated mice. Int. Immunopharmacol. 22 (1), 209-221. doi: 10.1016/j.intimp.2014.06.017

Sedgmen, B. J., Papalia, L., Wang, L., Dyson, A. R., McCallum, H. A., Simson, C. M., et al. (2013). Ex vivo restimulation of human PBMC expands a CD3 +CD4-CD8- gammadelta+ T cell population that can confound the evaluation of CD4 and CD8 T cell responses to vaccination. Clin. Dev. Immunol. 2013, 186420. doi: 10.1155/2013/186420 2013.

Strati, P., Wierda, W., Burger, J., Ferrajoli, A., Tam, C., Lerner, S., et al. (2013). Myelosuppression after frontline fludarabine, cyclophosphamide, and rituximab in patients with chronic lymphocytic leukemia: analysis of persistent and new-onset cytopenia. Cancer 119 (21), 3805-3811. doi: $10.1002 / \mathrm{cncr} .28318$

Tachi, T., Teramachi, H., Tanaka, K., Asano, S., Osawa, T., Kawashima, A., et al. (2015). The Impact of Outpatient Chemotherapy-Related Adverse Events on the Quality of Life of Breast Cancer Patients. PloS One 10 (4), e0124169. doi: 10.1371/journal.pone.0124169

Traverso, I., Fenoglio, D., Negrini, S., Parodi, A., Battaglia, F., Kalli, F., et al. (2012). Cyclophosphamide inhibits the generation and function of $\mathrm{CD} 8(+)$ regulatory T cells. Hum. Immunol. 73 (3), 207-213. doi: 10.1016/j.humimm.2011.12.020

Wang, J., Tong, X., Li, P., Cao, H., and Su, W. (2012). Immuno-enhancement effects of Shenqi Fuzheng Injection on cyclophosphamide-induced 
immunosuppression in Balb/c mice. J. Ethnopharmacol. 139 (3), 788-795. doi: 10.1016/j.jep.2011.12.019

Woo, S. M., Choi, Y. K., Kim, A. J., Yun, Y. J., Shin, Y. C., Cho, S. G., et al. (2016). Sip-jeon-dea-bo-tang, a traditional herbal medicine, ameliorates cisplatininduced anorexia via the activation of JAK1/STAT3-mediated leptin and IL6 production in the fat tissue of mice. Mol. Med. Rep. 13 (4), 2967-2972. doi: $10.3892 / \mathrm{mmr} .2016 .4889$

Zhang, M., Liu, X., Li, J., He, L., Qiu, M., and Tripathy, D. (2007). Chinese medicinal herbs to treat the side-effects of chemotherapy in breast cancer patients. Cochrane Database Syst. Rev. 18 (2), CD004921. doi: 10.1002/ 14651858.CD004921.pub2

Zhang, S., Zhou, L., Zhang, M., Wang, Y., Wang, M., Du, J., et al. (2019). Berberine Maintains the Neutrophil N1 Phenotype to Reverse Cancer Cell Resistance to Doxorubicin. Front. Pharmacol. 10, 1658. doi: 10.3389/ fphar.2019.01658
Zhao, N., Wang, L., Mou, H. Y., Liang, M., and Yue, W. (2009). [Synergism and attenuation effects of taurine on cyclophosphamide]. Ai Zheng 28 (3), 244-248. doi: 10.3321/j.issn:1000-467X.2009.03.004

Conflict of Interest: The authors declare that the research was conducted in the absence of any commercial or financial relationships that could be construed as a potential conflict of interest.

Copyright $\odot 2020$ Huang, Feng, Li, Zheng, Qi, Wang, Feng, Liu, Liu and Lu. This is an open-access article distributed under the terms of the Creative Commons Attribution License (CC BY). The use, distribution or reproduction in other forums is permitted, provided the original author(s) and the copyright owner(s) are credited and that the original publication in this journal is cited, in accordance with accepted academic practice. No use, distribution or reproduction is permitted which does not comply with these terms. 